\title{
Electronic and photoconductive properties of ultrathin InGaN photodetectors
}

Cite as: J. Appl. Phys. 103, 073715 (2008); https://doi.org/10.1063/1.2903146

Submitted: 11 December 2007 . Accepted: 25 January 2008 . Published Online: 09 April 2008

V. Lebedev, V. M. Polyakov, S. Hauguth-Frank, V. Cimalla, Ch. Y. Wang, G. Ecke, F. Schwierz, A. Schober, J. G. Lozano, F. M. Morales, D. González, O. Ambacher, et al.

\section{ARTICLES YOU MAY BE INTERESTED IN}

High-performance metal-semiconductor-metal InGaN photodetectors using as the insulator Applied Physics Letters 98, 103502 (2011); https://doi.org/10.1063/1.3562326

"S-shaped" temperature-dependent emission shift and carrier dynamics in InGaN/GaN multiple quantum wells

Applied Physics Letters 73, 1370 (1998); https://doi.org/10.1063/1.122164

Two dimensional electron gases induced by spontaneous and piezoelectric polarization in undoped and doped AlGaN/GaN heterostructures

Journal of Applied Physics 87, 334 (2000); https://doi.org/10.1063/1.371866

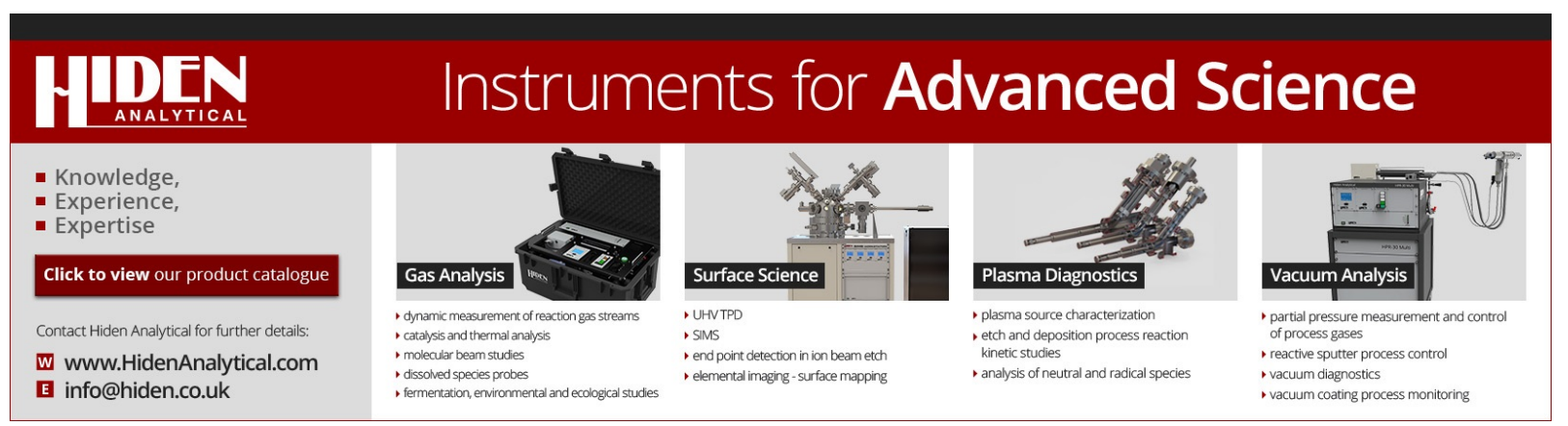




\title{
Electronic and photoconductive properties of ultrathin InGaN photodetectors
}

\author{
V. Lebedev, ${ }^{1, a)}$ V. M. Polyakov, ${ }^{1}$ S. Hauguth-Frank, ${ }^{1}$ V. Cimalla, ${ }^{1}$ Ch. Y. Wang, ${ }^{1}$ G. Ecke, ${ }^{1}$ \\ F. Schwierz, ${ }^{1}$ A. Schober, ${ }^{1}$ J. G. Lozano, ${ }^{2}$ F. M. Morales, ${ }^{2}$ D. González, ${ }^{2}$ and \\ O. Ambacher ${ }^{3}$ \\ ${ }^{1}$ Institute of Micro- and Nanotechnologies, Technical University Ilmenau, 98693 Ilmenau, Germany \\ ${ }^{2}$ Departamento de Ciencia de los Materiales e Ingeniería Metalúrgica y Química Inorgánica, \\ Facultad de Ciencias, Universidad de Cádiz, 11510 Puerto Real Cádiz, Spain \\ ${ }^{3}$ Fraunhofer Institute for Applied Solid State Physics, Tullastrasse 72, D-79108 Freiburg, Germany
}

(Received 11 December 2007; accepted 25 January 2008; published online 9 April 2008)

\begin{abstract}
We report on the compositional dependencies of electron transport and photoconductive properties for ultrathin metal-semiconductor-metal photodetectors based on In-rich $\operatorname{In}_{x} \mathrm{Ga}_{1-x} \mathrm{~N}$ alloys. For a $\mathrm{In}_{0.64} \mathrm{Ga}_{0.36} \mathrm{~N} / \mathrm{GaN}$ structure, the rise time close to the $R C$ constant at low fields has been measured along with a transparency of $\sim 77 \%$ and an absorbance of $\sim 0.2$ at a wavelength of $632 \mathrm{~nm}$. The electron density profiles and low-field mobilities for different compositions of InGaN have been calculated by numerically solving the Schrödinger and Poisson equations and applying the ensemble Monte Carlo method, respectively. It was demonstrated that in ultrathin $\operatorname{In}_{x} \mathrm{Ga}_{1-x} \mathrm{~N} / \mathrm{GaN}(0.5<x$ $<1$ ) heterostructures, in contrast to bulk InN exhibiting a strong surface electron accumulation, free electrons mostly tend to accumulate at the buried InGaN/GaN interface. We have also found that the low-field mobility in the InGaN/GaN heterostructures is strongly limited by the buried interface roughness which causes more than $95 \%$ of all scattering events occurred by two-dimensional electron transport under low electric field conditions. (C) 2008 American Institute of Physics.
\end{abstract}

[DOI: $10.1063 / 1.2903146]$

\section{INTRODUCTION}

The growing demand on high-precision instruments has led the research efforts toward the realization of specific devices for monitoring nanoscale motion. In particular, devices based on the concept of standing wave interferometry have been extensively investigated for this purpose. ${ }^{1}$ A key problem within these efforts is the development of a transparent ultrathin photodetector for sampling the intensity profile of the generated standing wave that recognize the movement of the object. Among the requirements for such phototodetector, transparency of $>70 \%$ at the $\mathrm{He}-\mathrm{Ne}$ laser wavelength $(\lambda=632 \mathrm{~nm})$, a low noise equivalent power, and cutoff frequencies of $>1 \mathrm{MHz}$ are essential to ensure the use in industrial applications. The previous attempts to develop an appropriate photodetector were based on either laterally ${ }^{2}$ or vertically ${ }^{3}$ arranged silicon photodiode concepts. However, they have been shown to be unsuited for industrial applications where long-term stability, reliability, and large dynamic range of measurable distances are key issues. Hence, it is worth moving toward the use of group III-nitride semiconductors with higher temperature stability and lower absorption coefficients at the wavelengths of interest. ${ }^{4}$ In particular, the use of $\operatorname{In}_{x} \mathrm{Ga}_{1-x} \mathrm{~N}$ thin films having direct band gaps ranging from $0.7(\mathrm{InN})$ to $3.5 \mathrm{eV}(\mathrm{GaN})$ is expected to increase both detector sensibility and reliability.

The major challenge related to $\mathrm{InN}$ and In-rich InGaN alloys is the reduction of electron concentration, which hinders, so far, the development of any kind of photoconducting

${ }^{\text {a)} E l e c t r o n i c ~ m a i l: ~ v a d i m . l e b e d e v @ i a f . f r a u n h o f e r . d e . ~}$ devices. Despite of the remarkable progress in the growth, thin epilayers appear to be degenerated and the mechanisms causing the degeneracy are still under debate. Additionally, the surfaces of $\mathrm{InN}$ and In-rich InGaN thin films exhibit an electron accumulation layer, with the surface Fermi level lying $0.9 \mathrm{eV}$ above the conduction band edge for InN. ${ }^{5-7}$ This is due to the extraordinary low conduction band minimum at the $\Gamma$-point, ${ }^{8}$ which allows donor-type surface states to be located inside the conduction band. In particular, for InN, a general model for the net carrier density $n_{e}$ has to include at least four independent components, ${ }^{9}$

$$
n_{e}=n_{\text {point }}+\frac{n_{L}+n_{i}}{d}+n_{e}^{\mathrm{TD}}
$$

where $d$ is the epilayer thickness, $n_{\text {point }} \sim 1 \times 10^{17} \mathrm{~cm}^{-3}$ is the background carrier density, which is the sum of intrinsic, impurity, and point-defect-generated carrier concentrations, $n_{L} \sim 3 \times 10^{13} \mathrm{~cm}^{-2}$ is the localized sheet carrier density at the surface, and $n_{e}^{\mathrm{TD}}$ and $n_{i}$ represent the dislocation generated and interface related electron densities, respectively. Moreover, for ultrathin epilayers, the contributions of $n_{i}$ and $n_{L}$ are at least comparable to the contribution of other generation mechanisms. ${ }^{10}$ This is also the case for In-rich $(x>0.5)$ $\mathrm{In}_{x} \mathrm{Ga}_{1-x} \mathrm{~N}$ alloys. For the latter, the surface Fermi-level position varies as a function of $x$ from high above the conduction band minimum for InN surfaces to significantly below this point for $\mathrm{GaN}$ surfaces, with the transition from electron accumulation to depletion occurring at approximately $x$ $\sim 0.3$. $^{11}$ Thus, exploitation of ultrathin $(d<\lambda / 4 \cdot n)$ In-rich InGaN photodetectors is only possible for a critical composition range where two conditions are fulfilled: (i) the signifi- 
cant decrease of the electron accumulation at the surface is occurred and (ii) a low bulk-related electron density $\left(<10^{17} \mathrm{~cm}^{-3}\right)$ is reached leading to a nondegenerated bulk electron continuum. Here, $d$ is the thickness of the detector layer and $n$ is the refractive index of the material. The electronic properties as well as the structural and optical quality of InGaN material strongly depend on the composition and thickness of the epilayers and are affected by strain, compositional fluctuations, and mismatch-induced dislocations, which have a deleterious effect on the detector performance.

In this work, we report on the electrical and optical characterization, as well as on the numerical simulation of electron transport properties of highly transparent metalsemiconductor-metal (MSM) $\operatorname{In}_{x} \mathrm{Ga}_{1-x} \mathrm{~N} / \mathrm{GaN}(0.56<x<1)$ photodetectors designed for standing-wave-interferometry at $\lambda=632 \mathrm{~nm}$. To evaluate the influence of the InGaN composition on the electronic properties of the epilayers, the electron density profiles and the low-field mobility of electrons have been calculated by means of self-consistent solution of the Schrödinger and Poisson equations and by using the ensemble Monte Carlo (MC) method, respectively. It is shown that in contrast to bulk $\mathrm{InN}$, a strong electron accumulation occurs at the buried InGaN/GaN interface, which is dictated by the presence of donorlike interface point defects in excess over the negative polarization-induced bound charge. This observation suggests that the transport properties of the twodimensional electron gas (2DEG) in the potential well located at the InGaN/GaN interface can be substantially influenced by the roughness of the buried interface.

\section{EXPERIMENTAL DETAILS}

The InGaN growth was carried out by plasma induced molecular beam epitaxy (PIMBE) using a GaN/AlN/ sapphire(0001) epitaxial template. The detailed description of the PIMBE experiments and characterization methods used can be found elsewhere. ${ }^{9,12}$ In this work, a series of $\sim 25 \mathrm{~nm}$ thick $\operatorname{In}_{x} \mathrm{Ga}_{1-x} \mathrm{~N}$ epilayers has been grown within the compositional range of $0.56<x<1$. The InGaN composition was determined by high resolution $\mathrm{x}$-ray diffraction (XRD). Finally, the choice of $\operatorname{In}_{0.64} \mathrm{Ga}_{0.36} \mathrm{~N}$ alloy as the detector layer was a compromise in order to obtain a welldetectable photoresponse and an absorption threshold located at $\lambda \sim 640 \mathrm{~nm}$ simultaneously.

Cross-section transmission electron microscopy (XTEM) experiments were performed in a JEM-2011 (JEOL) microscope operating at $200 \mathrm{kV}$. Selected area electron diffraction (SAED) patterns and micrographs obtained in bright field and dark field modes in conventional two-beam conditions were analyzed.

The MSM detector structures were fabricated by deposition of finger (type A) and rectangular (type B) Au/Ti contacts with spacings of $50 \mu \mathrm{m}$ and $0.5 \mathrm{~mm}$ corresponding to total series resistances $(R)$ of 27 and $600 \Omega$, respectively. The capacitance of the MSM detector (type B) of $C \sim 1 \mathrm{nF}$ was measured by means of a $L C R$ bridge at dark conditions at a frequency of $f=100 \mathrm{~Hz}$.

The single wavelength photoresponse has been measured by means of a lock-in technique using a $5 \mathrm{~mW} \mathrm{He}-\mathrm{Ne}$ laser.
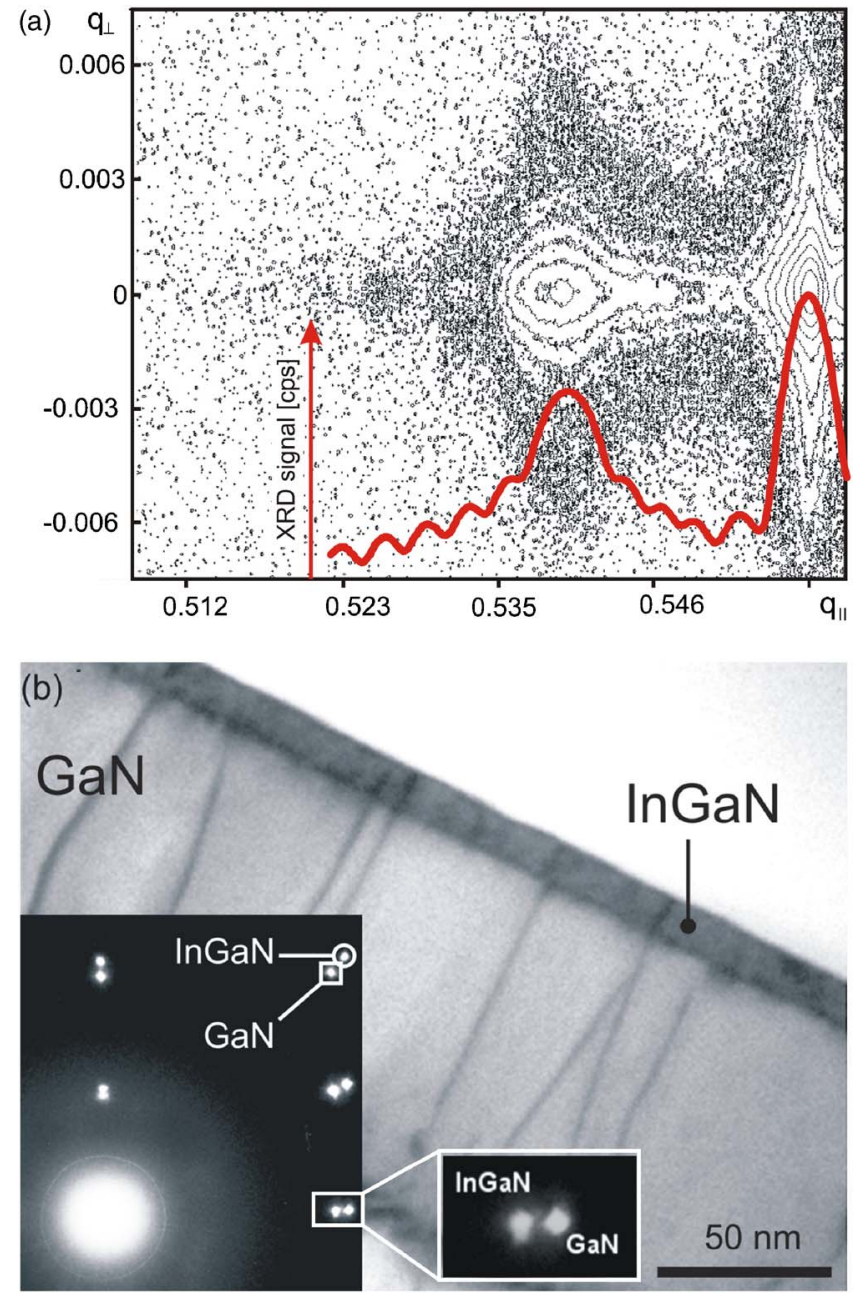

FIG. 1. (Color online) (Color online) (a) XRD RSM around the symmetric 0002 reflex of the $\operatorname{In}_{0.64} \mathrm{Ga}_{0.36} \mathrm{~N}$ epilayer grown on a $120 \mathrm{~nm}$ thick GaN template (inset: the XRD intensity oscillations around the 0002 reflex). (b) Bright-field XTEM micrograph taken under two beam conditions using the 0002 reflection near the $\langle 2-110\rangle$ zone axis. Inset: $\langle 2-1-110\rangle$ SAED pattern showing $\mathrm{GaN}$ and InGaN aligned reflection spots and a magnified image of the $(0-1-10)$ spot.

The light beam was modulated in the range of $10-10^{4} \mathrm{~Hz}$ using a mechanical chopper. The spectral response has been measured at a wavelength resolution of $\Delta \lambda \sim 2 \mathrm{~nm}$ in the range of $200-800 \mathrm{~nm}$ using a $150 \mathrm{~W}$ xenon-arc lamp, a single grid monochromator, and a lock-in technique at ambient temperature.

\section{RESULTS AND DISCUSSION}

\section{A. Structural and chemical properties}

For all InGaN samples, an almost fully relaxed $2 \mathrm{H}$-GaN(0001) layer was deposited as a buffer, typically $120 \mathrm{~nm}$ thick (see Fig. 1). For the $\mathrm{In}_{0.64} \mathrm{Ga}_{0.36} \mathrm{~N}$ layer, the XRD reciprocal space maps (RSMs) around the symmetric (0002) and the asymmetric (202-5) reflexes reveal the presence of in-plane stress [Fig. 1(a)]. In particular, a $c / a$ ratio of $\sim 1.672$ for $2 \mathrm{H}-\mathrm{In}_{0.64} \mathrm{Ga}_{0.36} \mathrm{~N}(0001)$ on $\mathrm{GaN}(0001)$ has been deduced (nominal $c / a \sim 1.601$ ) reflecting that a large biaxial strain of $\sim 1.3 \%$ built up in a $25 \mathrm{~nm}$ thick epilayer. The InGaN thickness was derived by fitting the XRD intensity 


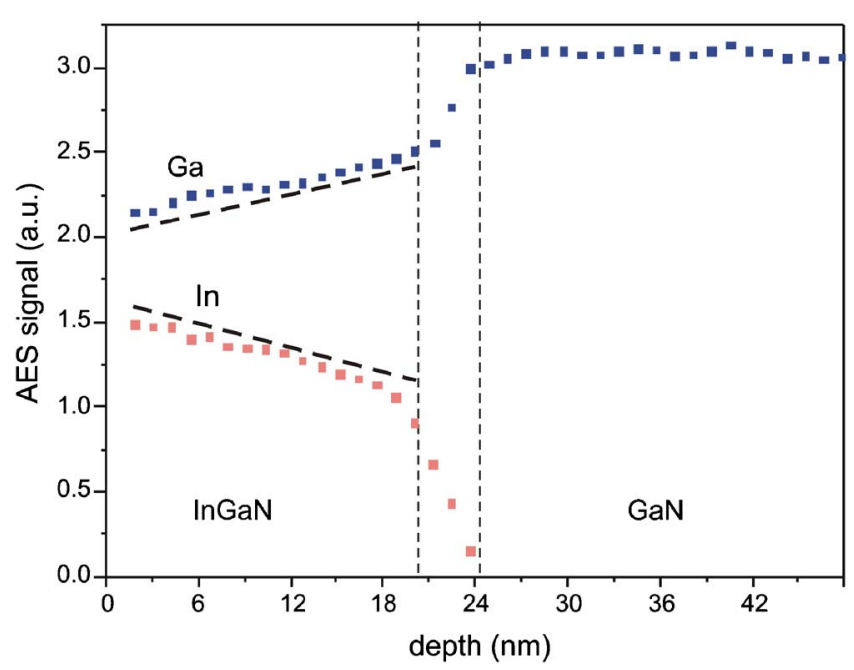

FIG. 2. (Color online) (Color online) AES depth profile of an InGaN/GaN heterostructure demonstrating the gradient in the composition of a $25 \mathrm{~nm}$ thick $\operatorname{In}_{0.64} \mathrm{Ga}_{0.36} \mathrm{~N}$ epilayer.

oscillations around the 0002 reflex using Diffrac LEPTOS software [see inset in Fig. 1(a)] and confirmed by XTEM measurements.

In Fig. 1(b), a characteristic bright field XTEM image using the $\mathbf{g}=0002$ reflection shows an $\mathrm{In}_{0.64} \mathrm{Ga}_{0.36} \mathrm{~N}$ epilayer grown on a $120 \mathrm{~nm}$ thick GaN template. The epilayer exhibits a homogeneous microstructure and a flat InGaN/GaN interface with little contrasts presented in the image. The SAED patterns shown in Fig. 1(b) present noncircular diffraction spots indicating InGaN compositional changes. ${ }^{13}$ The InGaN fundamental spots show an enlargement in the growth plane direction that implies the distortions in the $c$ lattice parameter. This fact is in agreement with a strong broadening of the 0002 reflex revealed by RSM. Typically, the well-known phase separation phenomena leading to the compositional modulation in InGaN thin films are observed within the growth plane. ${ }^{13-15}$ Nevertheless, the most probable explanation of such broadening is the formation of very thin striped areas with higher and lower $c$-lattice parameters revealing compositional changes where the in-plane $a$-lattice constants remained constant. This fluctuation in the In content along the $c$-axis can be explained by a thermodynamic process similar to spinodal decomposition driven by an enormous strain in the growing epilayer for these compositions. ${ }^{16}$ Gradual changes in the InGaN composition were also confirmed by Auger electron spectroscopy (AES) depth profiling shown in Fig. 2.

\section{B. Optical and temporal characteristics}

Optical transmission measurement of the photodetectors demonstrate a high transparency of $>70 \%$ with a contrast of $\sim 0.3 \%$ at $\lambda=632 \mathrm{~nm}$, which fits well the simulated optical data [see Fig. 3(a)]. The spectrally resolved photocurrent measurements show both an increase in the photoresponse at $\sim 1.86 \mathrm{eV}$ related to the band-to-band transition in the ultrathin $\mathrm{In}_{0.64} \mathrm{Ga}_{0.36} \mathrm{~N}$ epilayer as well as an absorption edge at $\sim 3.5 \mathrm{eV}$, which is related to the underlying GaN layer. At $632 \mathrm{~nm}$, the measured absorbance $(\alpha d)$ of a $25 \mathrm{~nm}$ thick
InGaN layer is more than ten times lower than that observed for the thicker GaN buffer. It is important to note that the gradual fluctuations of the In concentration discussed above lead to changes in the absorbance spectra of the photodetector, in particular, to a shift of the absorption edge towards lower photon energies.

The temporal response of the $\mathrm{In}_{0.64} \mathrm{Ga}_{0.36} \mathrm{~N} / \mathrm{GaN}$ MSM device (type B) at 5, 10, and $25 \mathrm{~V}$ applied bias is shown in Fig. 3(b). For the detectors studied, the rise time is limited mainly by the $R C$ time constant $\tau_{R C}$,

$$
\tau_{R C} \propto R\left(\varepsilon \varepsilon_{0} e n_{D} / V\right)^{1 / 2},
$$

where $R$ is the total resistance (depends mainly on the series resistance of the photodetector), $e$ is the elementary charge, $V$ is the bias, $\varepsilon \varepsilon_{0}$ is the dielectric permittivity of the InGaN alloy, and $n_{D}$ is the residual doping. A photoconductive time constant of $\sim 600 \mathrm{~ns}$ was evaluated at low fields due to the small series resistance coupled with a small capacitance.

The photoconductive decay is additionally affected by the pronounced persistent photocurrent (PPC) effect. ${ }^{12}$ The estimation of the decay constant $\tau_{0}$ for different biases was performed using a fit of the stretched-exponential function for the conductivity versus time,

$$
\sigma(t)=\sigma_{0}+b \exp \left\{-\left(t / \tau_{0}\right)^{\beta}\right\},
$$

where $0<\beta<1$ is the decay exponent, $\sigma_{0}$ and $b$ are constants, and $t$ is the time. A good fitting of the experimental data was obtained by this equation, showing that the faster part of the decay is actually limited by the $R C$ constant. The fitted values of $\tau_{0}$ are in the range of 500-600 ns corresponding to $\tau_{R C} \sim 600 \mathrm{~ns}$ and, therefore, do not depend significantly on the applied voltage in the low field regime.

Values of $\beta \sim 0.057-0.072$ indicate a slower part of the photoconductivity decay in form of an exponential tail caused by the PPC effect. This part furthermore shows a characteristic dependence on the biasing voltage. The slow response might be due to different physical processes, e.g., defect saturation as well as space-charge screening effects in the photoabsorbing layer. ${ }^{12}$ Indeed, if $\tau_{R C}$ is the only factor determining the time response, a significant decrease of time constants could be expected at high voltage in a trap-free semiconductor. If traps are present, the recharging processes change the kinetics of the electron-hole recombination, which is hindered by competitive retrapping of thermally released electrons causing a long time delay before the current reaches its steady dark level. The decay time constant for the slower decay part $\tau_{\mathrm{PPC}}$ is given by ${ }^{17}$

$$
\tau_{\mathrm{PPC}}^{-1}=\nu_{0} \exp \left(E_{t} / k T\right)
$$

where $\nu_{0} \sim 10^{11}-10^{12} \mathrm{~Hz}$ is the phonon-induced attempt to escape frequency and $E_{t} \sim 0.6-0.7 \mathrm{eV}$ is a rough estimation of the activation energy of the trap band obtained by the fit of Eq. (4) using the "slow decay" constant of $\sim 7 \mathrm{~s}$ derived from Eq. (3). ${ }^{4}$

The total response time $\tau_{\text {total }}$ can be expressed as

$$
\tau_{\text {total }}^{-1}=\tau_{\text {PPC }}^{-1}+\tau_{\text {drift }}^{-1}+\tau_{R C}^{-1},
$$

where $\tau_{\text {drift }}$ is the carrier drift time, which is on the order of picoseconds, and can be neglected. On the other hand, as 

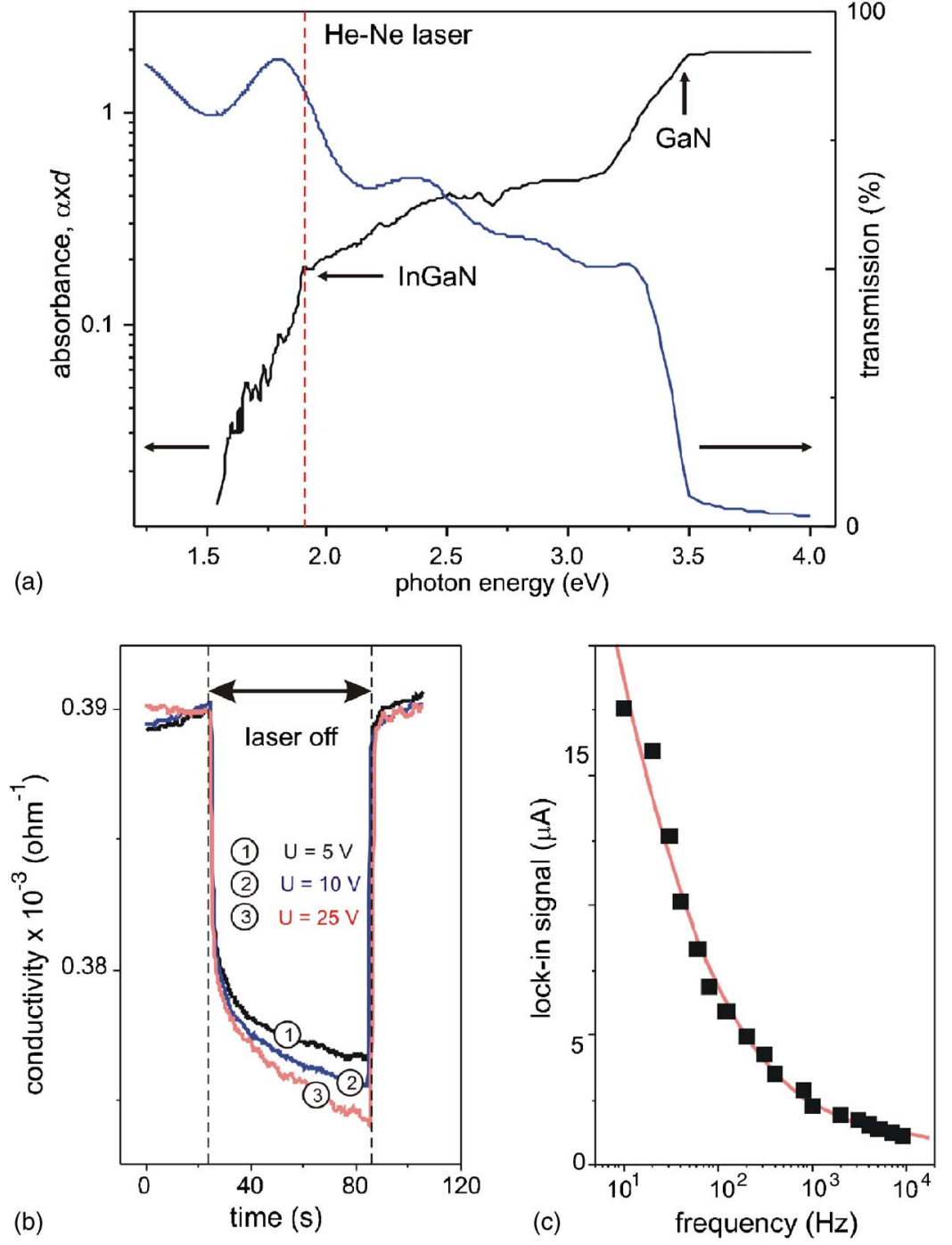

FIG. 3. (Color online) (Color online) (a) Absorbance, $\alpha d$, and optical transmission of the $\operatorname{In}_{0.64} \mathrm{Ga}_{0.36} \mathrm{~N} / \mathrm{GaN}$ photodetector obtained by transmission and spectrally resolved photocurrent methods. (b) Time-response measurement of the $\operatorname{In}_{0.64} \mathrm{Ga}_{0.36} \mathrm{~N}$ photodetector at 5, 10, and $25 \mathrm{~V}$ applied bias. (c) A lock-in signal ( $\sim$ peak photoresponse) at $632 \mathrm{~nm}$ as a function of the chopper frequency measured at $5 \mathrm{~V}$ bias. shown in Fig. 3(a), $\tau_{\text {PPC }}$ is a function of the applied electric field and increases with the bias voltage due to electron heating, lowering of the Coulomb-barrier ${ }^{17}$ and space-charge screening effect. ${ }^{12}$ Additionally, the contribution of a traprelated photoresponse from the underlying $\mathrm{GaN}$ epilayer at $\lambda=632 \mathrm{~nm}$ has to be taken into account. GaN-related PPC contributes to the observed $\tau_{s}$ versus bias dependence, which is due to the penetration of the electric field deeper into the GaN bulk causing more traps to be involved in the recharging processes.

In addition, a small increase of the photoconductive gain measured on the investigated ultrathin InGaN MSM detectors can be explained by the presence of structural defects and by the high electron densities, which both adversely affect the electron transport and the time response properties. The dependence between the photoresponse and the operational frequency, which is strongly influenced by the defect structure, is shown in Fig. 3(c), measured at $5 \mathrm{~V}$ applied bias by means of a $5 \mathrm{mV} \mathrm{He-Ne} \mathrm{laser} \mathrm{beam} \mathrm{and} \mathrm{the} \mathrm{lock-in} \mathrm{tech-}$ nique in the frequency range of $10-10^{4} \mathrm{~Hz}$. One can see that the spectral responsivity strongly depends on the frequency, mainly due to the deep carrier traps causing the PPC effect, similar to $\mathrm{AlGaN}$ photoconductors discussed elsewhere. ${ }^{12}$

\section{Electron transport properties}

In Fig. 4, the compositional dependence of the Hall sheet electron concentration $n_{H}=n_{e}(x) d$ and mobility $\mu(x)$ determined for a series of $25 \mathrm{~nm}$ thick $\operatorname{In}_{x} \mathrm{Ga}_{1-x} \mathrm{~N}$ films with $x$ ranging from 1 to 0.56 are shown. For ultrathin In-rich In$\mathrm{GaN}$ alloys grown on GaN, $n_{e} d$ represents the sum of $n_{L}, n_{i}$, and $n_{e}^{\mathrm{TD}} d$ being mainly a function of crystal quality and composition $x$. Firstly, we can assume that $n_{e}^{\mathrm{TD}}(x) d$ is a weak function of composition and kept approximately constant through the investigated range of compositions. In this case, the observed reduction of $n_{e}(x) d$ can be related to the decrease of the surface $\left[n_{L}(x)\right]$ and interface $\left[n_{i}(x)\right]$ accumulated charge, which depend on the downward band bending at the InGaN surface and positive polarization bound charge at the buried $\mathrm{InGaN} / \mathrm{GaN}$ interface, respectively.

The compositional dependence of the separation between the conduction band minimum and the surface Fermi level $\Phi_{B}$ has been estimated empirically by Veal and co-workers ${ }^{11}$ for thick $\operatorname{In}_{x} \mathrm{Ga}_{1-x} \mathrm{~N}$ films suggesting that $\Phi_{B}=0$ at $x \sim 0.3$. The transition from surface electron depletion to accumulation additionally depends on the position of the surface Fermi level with respect to the bulk Fermi level. In particular, $\operatorname{In}_{x} \mathrm{Ga}_{1-x} \mathrm{~N}$ samples with $x<0.43$ exhibiting no 


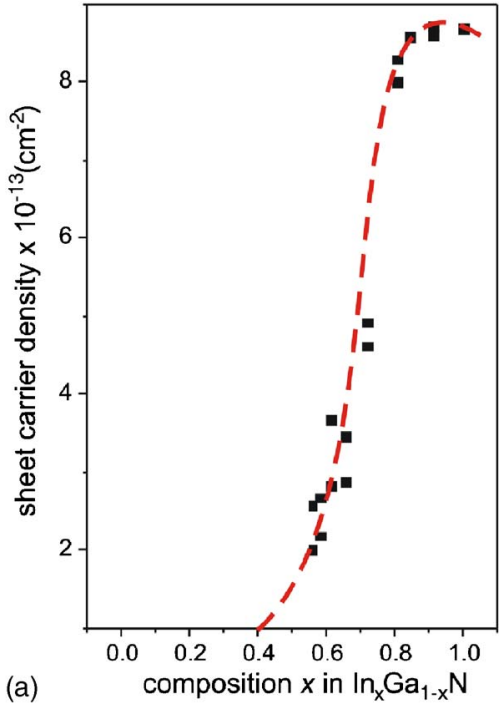

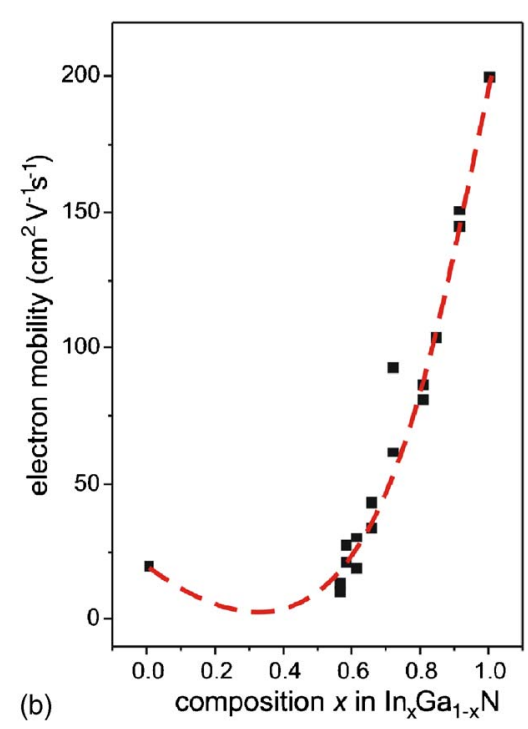

FIG. 4. (Color online) (Color online) (a) Hall sheet carrier density and (b) electron mobility for the series of $25 \mathrm{~nm}$ thick $\mathrm{In}_{x} \mathrm{Ga}_{1-x} \mathrm{~N}$ films as a function of In concentration $x$. surface accumulation have been reported. ${ }^{18}$ These observations are partly consistent with our data derived from Hall [Fig. 4(a)] and photocurrent measurements. The observed variation of the sheet carrier density with $x$ shows that at $x \sim 0.7$ a pronounced decrease of $n_{e}$ occurs indicating that the surface induced gap states change their charging character from donor to acceptor type. However, no InGaN-related photoresponse was observed suggesting that the bulk Fermi level is still located within the conduction band. An obvious InGaN photoresponse was observed at $x<0.64$ manifesting nondegenerated bulk electrons and a significantly reduced surface electron accumulation. The latter becomes evident from the experiments on an ozone-assisted oxidation of $\mathrm{In}_{0.64} \mathrm{Ga}_{0.36} \mathrm{~N}$ samples. ${ }^{18,19}$ It was observed that the oxidation treatment results in a weak reduction of the sheet carrier density of $\Delta n_{e} \sim-2 \times 10^{12} \mathrm{~cm}^{-2}$ and a simultaneous small increase of the electron mobility of $\Delta \mu \sim 2 \mathrm{~cm}^{2} \mathrm{~V}^{-1} \mathrm{~s}^{-1}$. These findings indicate the presence of the surface accumulated electron charge, which can be manipulated by a passivation of the surface donors.

Next, the composition dependence of $\mu(x)$ [Fig. 4(b)] shows a sharp decrease of the electron mobility from $\sim 200 \mathrm{~cm}^{2} \mathrm{~V}^{-1} \mathrm{~s}^{-1}$ (for $\sim 25 \mathrm{~nm}$ thick $\mathrm{InN}$ ) down to below the experimentally measured mobility for a $30 \mathrm{~nm}$ thick GaN layer. This reduction of the mobility cannot be ascribed only to increasing electron effective mass varying from $0.04 m_{0}$ $(\mathrm{InN})$ to $0.20 m_{0}(\mathrm{GaN})$. To explain such compositional dependence of the electron mobility, an additional scattering mechanism, namely, due to the buried interface roughness, has to be invoked as discussed in the following section.

\section{Calculation of the electron density depth profiles and low-field mobilities}

To evaluate the influence of In content on the electronic properties of $\operatorname{In}_{x} \mathrm{Ga}_{1-x} \mathrm{~N} / \mathrm{GaN}$ heterosystem, the electron density depth distribution and the low-field mobility of the carriers in InGaN layer have been calculated by selfconsistently solving the Schrödinger and Poisson equations and by using the ensemble MC method, respectively. ${ }^{20} \mathrm{Ma}-$ terial and band structure parameters of ternary InGaN for various values of In content ranging from 0.5 to 1.0 have been obtained through the linear interpolation between binary InN and GaN constituents.

Due to a finite barrier height of the $\mathrm{InGaN} / \mathrm{GaN}$ heterojunction, by solving the Schrödinger equation, the electron wave functions were allowed to penetrate into GaN substrate. The downward surface band bending of $-0.9 \mathrm{eV}$ for $x=1.0$ and the surface flat band conditions (zero band bending) $)^{11}$ for $x=0.3$ have been assumed. For In contents ranging from $x=0.5$ to $x=1.0$, the surface band bending was linearly interpolated. Ideally, in pseudomorphically grown InGaN/GaN heterostructures, a negative polarization bound charge is formed at the buried interface. ${ }^{21}$ However, as follows from our calculations, the net interface charge has to be positive in order to theoretically reproduce the measured sheet densities. Accordingly, we have to assume the presence of donorlike interface point defects which substantially overcompensate the negative polarization-induced charge. Finally, this positive net interface charge $\sigma_{\text {net }}$ was considered as a fitting parameter to match the calculated and measured electron sheet densities (see Table I). Figure 5 presents the compositional dependence of the electron sheet density calculated by self-consistently solving the Poisson and Schrödinger equations in comparison to the measured values.

The calculated electron density depth profiles for $x=0.5$ and $x=1.0$ are depicted in Fig. 6. Interestingly, in

TABLE I. Values of the net interface charge $\sigma_{\text {net }}$ and interface roughness $\Delta$ used in the calculations for fitting the measured electron sheet densities and low-field mobilities.

\begin{tabular}{ccc}
\hline \hline In content & $\begin{array}{c}\text { Net interface } \\
\text { charge } \\
\left(\sigma_{\text {net }} / \mathrm{q}\right) \times 10^{-13} \\
\mathrm{~cm}^{-2}\end{array}$ & \\
\hline 0.5 & 1.1 & 5.6 \\
0.6 & 1.7 & 3.0 \\
0.7 & 3.5 & 1.2 \\
0.8 & 6.9 & 0.53 \\
0.9 & 7.4 & 0.45 \\
1.0 & 7.5 & 0.55 \\
\hline \hline
\end{tabular}




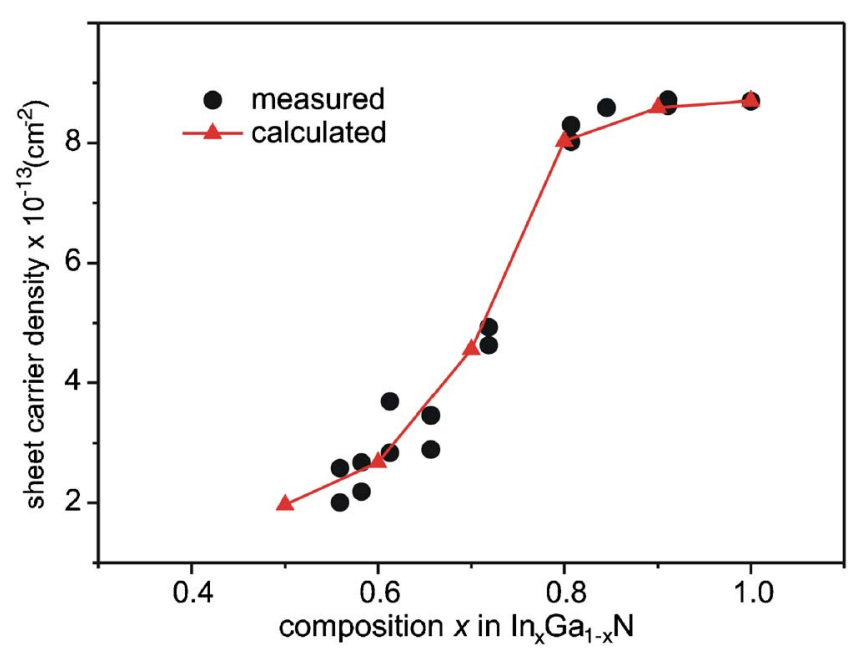

FIG. 5. (Color online) (Color online) Compositional dependence of the calculated and measured electron sheet densities in $\operatorname{In}_{x} \mathrm{Ga}_{1-x} \mathrm{~N} / \mathrm{GaN}$ heterostructures.

contrast to the dominating surface accumulation characteristic for bulk InN, in the case of thin InGaN epilayers grown on $\mathrm{GaN}$, free electrons tend mostly to accumulate in the vicinity to the buried interface. The latter, as already mentioned above, is accounted for by the presence of donorlike defects at the interface. ${ }^{9,22,23}$ It is worth mentioning that the dislocation-derived $n$-type doping was also accounted for when solving the Schrödinger and Poisson equations. We assumed that the dislocation density is $N_{\text {disl }}=2.85$ $\times 10^{11} \mathrm{~cm}^{-2}$ and the donor state (one donor per unit length along $c$-axis) is resonant with the conduction band at $E_{D}$ $=100 \mathrm{meV}$. However, as indicated in Fig. 6, the dislocationderived donors in the region close to the interface are fully neutralized since the donor level falls well below the Fermi energy $E_{F}=0$. Consequently, the dislocations cannot remarkably influence the electron mobility. This observation suggests that transport properties of the 2DEG in the InGaN/GaN potential well have to be mostly influenced by the buried interface roughness, even to a larger extent if compared to surface roughness scattering, which takes place in case of bulk InN. ${ }^{20}$

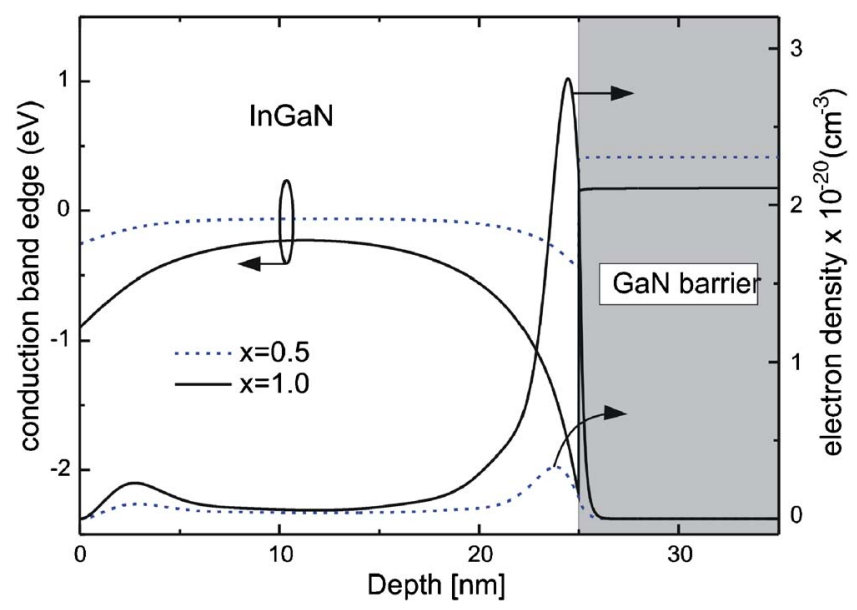

FIG. 6. (Color online) (Color online) Depth profiles of the conduction band and the electron density for two compositions $x=0.5$ and $x=1.0$ of $\mathrm{In}_{x} \mathrm{Ga}_{1-x} \mathrm{~N} / \mathrm{GaN}$ heterostructures.

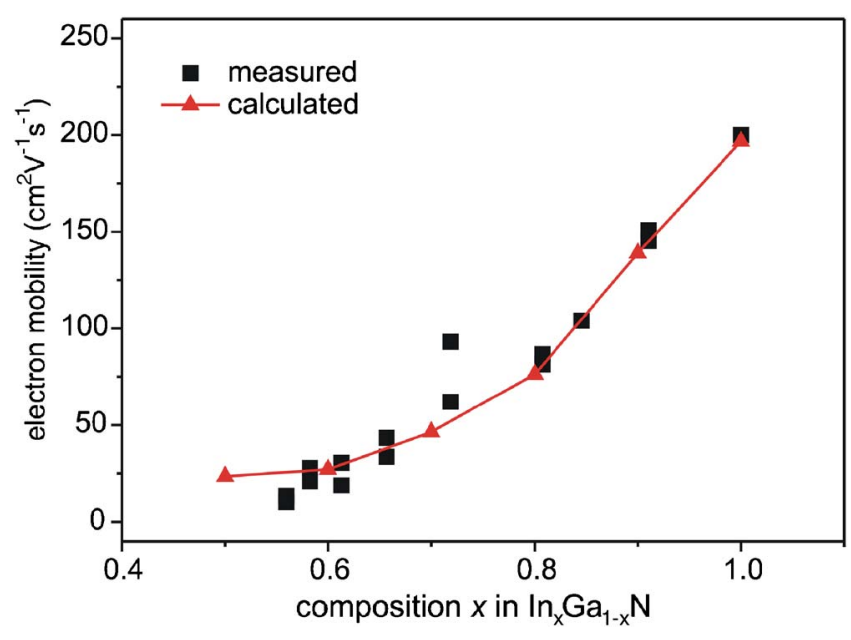

FIG. 7. (Color online) (Color online) Compositional dependence of the calculated and measured low-field mobilities in $25 \mathrm{~nm}$ thick $\operatorname{In}_{x} \mathrm{Ga}_{1-x} \mathrm{~N}$ epilayer on $\mathrm{GaN}$.

To validate these expectations, we applied the ensemble MC method for the calculation of the low-field mobility of InGaN/GaN heterostructure as a function of In content. Our approach is similar to that applied in our previous work. ${ }^{20}$ Here, however, we consider the interface roughness as additional scattering mechanism independent from the surface roughness, i.e., the surface and buried interface roughnesses are assumed to be uncorrelated. As the electron wave functions are allowed to penetrate into $\mathrm{GaN}$ substrate, we also account for a finite barrier height of $\mathrm{InGaN} / \mathrm{GaN}$ heterojunction that can substantially modify the interface roughness scattering rate. ${ }^{24}$ To characterize the surface/interface roughness, parameters such as the average height of terraces presented at the surface or/and interface $\Delta$ and the correlation length $L$ (average length of terraces) were used. Other details of our theoretical model including scattering mechanisms due to phonons and charged edge dislocations can be found elsewhere. ${ }^{20}$ The results of the low-field mobility calculation are plotted in Fig. 7. It was found that the low-field mobility is mostly limited by the buried interface roughness which causes more than $95 \%$ of all scattering events occurring in the 2DEG under low electric field conditions (less than $1 \mathrm{kV} / \mathrm{cm})$.

Further, it should be noted that to get a better agreement to the measured mobilities for different compositions, we assumed an increasing interface roughness $\Delta$ for decreasing In content in $\mathrm{In}_{x} \mathrm{Ga}_{1-x} \mathrm{~N}$ (see Table $\mathrm{I}$ ). This tendency taken into account in our MC calculations can be ascribed to the presence of composition modulations experimentally observed in In-rich $\operatorname{In}_{x} \mathrm{Ga}_{1-x} \mathrm{~N}$ ternary compounds close to the interface. ${ }^{16,25}$ Then, such modulations are taken into account by effectively increasing the roughness parameter $\Delta$ in our rather phenomenological model of the interface roughness scattering.

\section{CONCLUSIONS}

In conclusion, the compositional dependencies of electron transport properties, photoresponse as well as temporal characteristics have been studied for ultrathin MSM struc- 
tures based on In-rich $\operatorname{In}_{x} \mathrm{Ga}_{1-x} \mathrm{~N}$ alloys grown by PIMBE on GaN epitaxial templates. For the $\operatorname{In}_{0.64} \mathrm{Ga}_{0.36} \mathrm{~N} / \mathrm{GaN}$ detector, a rise time close to the $R C$ constant at low fields has been measured, along with a transparency of more than $77 \%$ and an absorbance of $\sim 0.2$ at a wavelength of $632 \mathrm{~nm}$. Structural analyses on an $\mathrm{In}_{0.64} \mathrm{Ga}_{0.3} 6 \mathrm{~N}$ layer carried out by high resolution XRD and XTEM have ascertained a large biaxial strain of $\sim 1.3 \%$. A strong broadening of the 0002 reflex implying a gradual change in the In content along the $c$-axis forced by enormous strain in the growing epilayer is observed.

By self-consistently solving the Schrödinger and Poisson equations, it was shown that in contrast to the strong surface electron accumulation characteristic for bulk InN, ultrathin $\mathrm{In}_{x} \mathrm{Ga}_{1-x} \mathrm{~N} / \mathrm{GaN} \quad(0.5<x<1)$ heterostructures exhibit a dominating electron accumulation at the buried $\mathrm{InGaN} / \mathrm{GaN}$ interface due to the presence of donorlike interface defects. Using the ensemble MC method, it was demonstrated that the low-field mobility of the $2 \mathrm{DEG}$ in such epilayers is mostly limited by the buried interface roughness whereas the dislocation scattering is almost fully suppressed due to the neutralization of the dislocation-associated donors in the vicinity to the interface.

\section{ACKNOWLEDGMENTS}

This work was partially supported by CICYT project MAT2007-60643 (Spain), Arbeitsgemeinschaft industrielle Forschungsvereinigungen (Germany) and Deutsche Forschungsgemeinschaft (Germany).

${ }^{1}$ E. Bunte, V. Mandryka, K. H. Jun, H.-J. Büchner, G. Jäger, and H. Stiebig, Sens. Actuators, A 113, 334 (2004).

${ }^{2}$ M. Sasaki, X. Mi, and K. Hahne, J. Phys. Soc. Jpn. 48, 55 (2001).

${ }^{3}$ H. Stiebig, H. Büchner, and E. Bunte, Appl. Phys. Lett. 83, 12 (2003).

${ }^{4}$ G. Mazzeo, G. Conte, J.-L. Reverchon, A. Dussaigne, and J.-Y. Duboz, Appl. Phys. Lett. 89, 223513 (2006).
${ }^{5}$ H. Lüth, Phys. Status Solidi A 187, 33 (2001).

${ }^{6}$ H. Lu, W. J. Schaff, L. F. Eastman, and C. E. Stutz, Appl. Phys. Lett. 82, 1736 (2003)

${ }^{7}$ V. Cimalla, M. Niebelschütz, G. Ecke, V. Lebedev, O. Ambacher, M. Himmerlich, S. Krischok, J. A. Schaefer, H. Lu, and W. Schaff, Phys. Status Solidi A 203, 59 (2006).

${ }^{8}$ I. Mahboob, T. D. Veal, L. F. J. Piper, C. F. McConville, H. Lu, W. J. Schaff, J. Furthmüller, and F. Bechstedt, Phys. Rev. B 69, 201307 (2004).

${ }^{9}$ V. Lebedev, V. Cimalla, T. Baumann, O. Ambacher, F. M. Morales, J. G. Lozano, and D. González, J. Appl. Phys. 100, 094903 (2006).

${ }^{10}$ V. Cimalla, V. Lebedev, F. M. Morales, R. Goldhahn, and O. Ambacher, Appl. Phys. Lett. 89, 172109 (2006).

${ }^{11}$ T. D. Veal, P. H. Jefferson, L. F. J. Piper, C. F. McConville, T. B. Joyce, P. R. Chalker, L. Considine, H. Lu, and W. J. Schaff, Appl. Phys. Lett. 89, 202110 (2006); P. D. C. King, T. D. Veal, P. H. Jefferson, C. F. McConville, H. Lu, and W. J. Schaff, Phys. Rev. B 75, 115312 (2007).

${ }^{12}$ V. Lebedev, G. Cherkashinin, G. Ecke, I. Cimalla, and O. Ambacher, J. Appl. Phys. 101, 033705 (2007).

${ }^{13}$ A. N. Westmeyer and S. Mahajan, Appl. Phys. Lett. 79, 2710 (2001).

${ }^{14}$ M. Rao, D. Kim, and S. Mahajan, Appl. Phys. Lett. 85, 1961 (2004).

${ }^{15}$ T. Bartel, J. R. Jinschek, B. Freitag, P. Specht, and C. Kisielowski, Phys. Status Solidi A 203, 167 (2006).

${ }^{16}$ T. Bartel, P. Specht, J. C. Hoz, and C. Kisielowski, Philos. Mag. 87, 1983 (2007).

${ }^{17}$ R. H. Bube, Photoelectronic Properties of Semiconductors (Cambridge University Press, New York, 1992), p. 45.

${ }^{18}$ V. Cimalla, V. Lebedev, Ch. Y. Wang, M. Ali, G. Ecke, V. M. Polyakov, F. Schwierz, and O. Ambacher, Appl. Phys. Lett. 90, 152106 (2007).

${ }^{19}$ V. Lebedev, Ch. Y. Wang, V. Cimalla, S. Hauguth, T. Kups, M. Ali, G. Ecke, M. Himmerlich, S. Krischok, J. A. Schaefer, O. Ambacher, V. M. Polyakov, and F. Schwierz, J. Appl. Phys. 101, 123705 (2007).

${ }^{20}$ V. M. Polyakov and F. Schwierz, J. Appl. Phys. 101, 033703 (2007).

${ }^{21}$ O. Ambacher, J. Smart, J. R. Shealy, N. G. Weimann, K. Chu, M. Murphy, W. J. Schaff, L. F. Eastman, R. Dimitrov, L. Wittner, M. Stutzmann, W. Rieger, and J. Hilsenbeck, J. Appl. Phys. 85, 3222 (1999).

${ }^{22}$ T. D. Veal, L. F. J. Piper, I. Mahboob, H. Lu, W. J. Schaff, and C. F. McConville, Phys. Status Solidi C 2, 2246 (2005).

${ }^{23}$ K. Wang, C. Lian, N. Su, D. Jena, and J. Timler, Appl. Phys. Lett. 91, 232117 (2007).

${ }^{24}$ P. J. Price and F. Stern, Surf. Sci. 132, 577 (1983).

${ }^{25}$ A. Vantomme, M. Fang Wu, S. Hogg, G. Langouche, K. Jacobs, I. Moerman, M. White, K. O'Donnell, L. Nistor, J. Van Landuyt, and H. Bender, MRS Internet J. Nitride Semicond. Res. 5S1, W11.38 (2000). 